\title{
The topology of the external activity complex of a matroid
}

\author{
Federico Ardila* \\ Department of Mathematics \\ San Francisco State University \\ San Francisco, CA, U.S.A. \\ federico@sfsu.edu
}

\author{
Federico Castillo \\ Department of Mathematics \\ University of California, Davis \\ Davis, CA, U.S.A. \\ fcastillo@math.ucdavis.edu
}

\author{
José Alejandro Samper \\ Department of Mathematics \\ University of Washington \\ Seattle, WA, U.S.A. \\ samper@math. washington.edu
}

Submitted: Feb 13, 2015; Accepted: Jul 4, 2016; Published: Jul 22, 2016

Mathematics Subject Classifications: 05C88, 05C89

\begin{abstract}
We prove that the external activity complex $\operatorname{Act}_{<}(M)$ of a matroid is shellable. In fact, we show that every linear extension of LasVergnas's external/internal order $<_{\text {ext/int }}$ on $M$ provides a shelling of $\operatorname{Act}_{<}(M)$. We also show that every linear extension of LasVergnas's internal order $<_{i n t}$ on $M$ provides a shelling of the independence complex $I N(M)$. As a corollary, $\operatorname{Act}_{<}(M)$ and $M$ have the same $h$ vector. We prove that, after removing its cone points, the external activity complex is contractible if $M$ contains $U_{1,3}$ as a minor, and a sphere otherwise.
\end{abstract}

\section{Introduction}

Wider context of this work. Matroid theory is a combinatorial theory of independence which has its roots in linear algebra and graph theory, but which turns out to have deep connections with many fields. There are natural notions of independence in linear algebra, graph theory, matching theory, the theory of field extensions, and the theory of routings, among others. Matroids capture the combinatorial essence that those notions share.

A matroid can be described in many equivalent ways, arising from the many contexts where matroids are found: the bases, the circuits, the lattice of flats, and the matroid polytope, among others. One important approach, which is the most relevant one to this paper, has been to model a matroid in terms of a simplicial or polyhedral complex. In fact,

*Partially supported by the US National Science Foundation CAREER Award DMS-0956178 and the SFSU-Colombia Combinatorics Initiative. 
most of these topological models arise naturally in algebraic and geometric contexts, and offer new tools to prove combinatorial theorems. A celebrated recent example is the proof by Huh [10] and Huh and Katz [11] of Rota's 1971 conjecture [22] that the coefficients of the characteristic polynomial of a linear matroid are unimodal. A key ingredient of this proof is the Bergman complex $\mathcal{B}(M)$ described below.

Let us describe a few constructions of this flavor, and provide a few references for the interested reader. The notion of shellability is a very useful unifying tool in this approach, as explained in [4].

- [20,4] The independence complex or matroid complex $I N(M)$ is homotopy equivalent to a wedge of $T_{M}(0,1)=\left|\mu\left(M^{*}\right)\right|$ spheres of dimension $r(M)-1$ if $M$ is coloopless. This complex is shellable, and its shelling polynomial is $T_{M}(x, 1)$. The shellability of $I N(M)$ naturally leads to the important notions of internal and external activity of $M$.

- $[27,21,6]$ The broken circuit complex $\overline{B C}_{<}(M)$ is, a cone over a space homotopy equivalent to a wedge of $|\beta(M)|$ spheres of dimension $r(M)-2$. It can be naturally embedded into $I N(M)$. It is shellable and its shelling polynomial is $T_{M}(x, 0)$. The embedding is a combinatorial witness of such a result. Its face numbers equal the coefficients of the characteristic polynomial of $M$ up to sign.

- $[9,24,18]$ the (proper part of the) order complex of the lattice of flats $\Delta\left(L_{M} \backslash\{\widehat{0}, \widehat{1}\}\right)$ is homotopy equivalent to a wedge of $T_{M}(1,0)=|\mu(M)|$ spheres of dimension $r(M)-2$. It is shellable. This is a motivating example for the theory of Cohen Macaulay posets. It also arises naturally in Orlik and Solomon's presentation of the cohomology of the complement of a complex hyperplane arrangement.

- $[25,3]$ The Bergman complex $\mathcal{B}(M)$ is the link of the origin in the tropical linear space $\operatorname{Trop}(M)$. It is not always simplicial. Though not obvious from its definition, $\mathcal{B}(M)$ is a coarsening of $\Delta\left(L_{M} \backslash\{\widehat{0}, \widehat{1}\}\right)$ and hence shares its topological properties. These complexes are fundamental objects in tropical geometry because $\operatorname{Trop}(M)$ is the tropical analog of a linear space.

The purpose of this paper is to describe a new member of this family.

- The external activity complex $\operatorname{Act}_{<}(M)$ is, after removing cone points, either contractible or a sphere of dimension $n+r-1-|A E(M)|$ where $A E(M)$ is the set of externally absolute elements. It contains a copy of $I N(M)$ as a subcomplex. It is shellable, and its shelling polynomial is $T_{M}(x, 1)$. Its shellability is closely related to Las Vergnas's active orders on the bases of $M$.

So the external activity complex sheds new light on the shelling polynomial $T_{M}(x, 1)$ of a matroid $M$. This is a subject of great attention thanks to Stanley's 1977 h-vector conjecture, one of the most intriguing open problems in matroid theory:

Conjecture 1.1. [24] For any matroid $M$, there exists a set $X$ of monomials such that:

- if $m$ and $m^{\prime}$ are monomials such that $m \in X$ and $m^{\prime} \mid m$, then $m^{\prime} \in X$,

- all the maximal monomials in $X$ have the same degree,

- there are exactly $h_{i}$ monomials of degree $i$ in $X$, where $\sum_{i} h_{i} x^{r-i}=T(x, 1)$.

This conjecture has been proved, using rather different methods, for several families: cographic matroids, [15], lattice path matroids [23], cotransversal matroids [17], paving matroids [16], and matroids up to rank 4 or corank $2[8,12]$. The general case remains open. 
Motivation for this work. The external activity complex $\operatorname{Act}_{<}(M)$ of a matroid is a simplicial complex associated to a matroid $M$ and a linear order $<$ on its ground set. This complex arose in work of the first author with Adam Boocher [2]. They started with a linear subspace $L$ of affine space $\mathbb{A}^{n}$ with a chosen system of coordinates. There is a natural embedding $\mathbb{A}^{n} \hookrightarrow\left(\mathbb{P}^{1}\right)^{n}$ into a product of projective lines, and they considered the closure $\widetilde{L}$ of $L$ in $\left(\mathbb{P}^{1}\right)^{n}$. They proved that many geometric and algebraic invariants of the variety $\widetilde{L}$ are determined by the matroid of $L$.

As is common in combinatorial commutative algebra, a key ingredient of [2] was to consider the initial ideals in $\widetilde{L}_{<}$under various term orders. These initial ideals are the Stanley-Reisner ideals of the external activity complexes $\operatorname{Act}_{<}(M)$ under the different linear orders $<$ of the ground set. This led them to consider and describe the complexes $\operatorname{Act}_{<}(M)$.

The ideals in $_{<} \widetilde{L}$ are shown to be Cohen-Macaulay in [2], and the authors asked the stronger question: Are the external activity complexes $\operatorname{Act}_{<}(M)$ shellable? The purpose of this note is to answer this question affirmatively.

Our results. The facets of $\operatorname{Act}_{<}(M)$ are indexed by the bases $\mathcal{B}$ of $M$, and [2] suggested a possible connection between $\operatorname{Act}_{<}(M)$ and LasVergnas's internal order $<_{\text {int }}$ on $\mathcal{B}$. Suprisingly, we find that it is the external/internal order $<_{\text {ext }}$ int on $\mathcal{B}$, also defined in [14], which plays a key role. Our main result is the following:

Theorem 1.2. Let $M=(E, \mathcal{B})$ be a matroid, and let $<$ be a linear order on the ground set $E$. Any linear extension of LasVergnas's external/internal order $<_{\text {ext } / \text { int }}$ of $\mathcal{B}$ induces a shelling of the external activity complex $\operatorname{Act}_{<}(M)$.

As a corollary we obtain that these orders also shell the independence complex $I N(M)$, and in fact we show a stronger statement.

Theorem 1.3. Any linear extension of the internal order $<_{\text {int }}$ gives a shelling order of the independence complex $I N(M)$.

These theorems are as strong as possible in the context of LasVergnas's active orders. We also obtain the following enumerative corollary.

Theorem 1.4. The $h$-vector of $\operatorname{Act}_{<}(M)$ equals the $h$-vector of $M$.

It is easy to see that $\operatorname{Act}_{<}(M)$ is a cone, and hence trivially contractible. It is more interesting to study the reduced external activity complex $\operatorname{Act}_{<}^{\bullet}(M)$, obtained by removing all the cone points of $\operatorname{Act}_{<}(M)$. Our main topological result is the following.

Theorem 1.5. Let $M$ be a matroid and < be a linear order on its ground set. The reduced external activity complex $\operatorname{Act}_{<}^{\bullet}(M)$ is contractible if $M$ contains $U_{1,3}$ as a minor, and a sphere otherwise.

In Proposition 6.5 we will see there is an embedding of the independence complex $I N(M)$ in $\operatorname{Act}_{<}^{\bullet}(M)$, and both complexes have the same $h$-vector. If $M$ is coloopless its independence complex is homotopy equivalent to a wedge of $\left|\mu\left(M^{*}\right)\right|$ spheres, while the external activity complex is contractible or a sphere. Thus $\operatorname{Act}_{<}^{\bullet}(M)$ can be seen as a topologically simpler model than $I N(M)$ for the matroid $M$. 
The paper is organized as follows. In Section 2 we introduce the necessary definitions and preliminaries. In Section 3 we carry out an example in detail, and show that the hypotheses of Theorems 1.2 and 1.3 are best possible. In Section 4 we prove our main Theorem 1.2 on the shellability of the external activity complex $\operatorname{Act}_{<}(M)$, and Theorem 1.3, which gives many new shellings of the independence complex $I N(M)$. In Section 5 we show that $\operatorname{Act}_{<}(M)$ and $I N(M)$ have the same $h$-vector. Finally, in Section 6, we describe the topology of the reduced external activity complex in Theorem 1.5.

\section{Preliminaries}

In this section we collect the background information on matroids and shellability that we will need to prove our results.

\subsection{Matroids}

Basic definitions. A simplicial complex $\Delta=(E, \mathcal{I})$ is a pair where $E$ is a finite set and $\mathcal{I}$ is a non empty family of subsets of $E$, such that if $A \in \mathcal{I}$ and $B \subset A$, then $B \in A$. Elements of $\mathcal{I}$ are called faces of the complex. The maximal elements of $\mathcal{I}$ are called facets. A complex is said to be pure if all facets have the same number of elements.

The following is one of many equivalent ways of defining a matroid:

Definition 2.1. A matroid $M=(E, \mathcal{I})$ is a simplicial complex such that the restriction of $M$ to any subset of $E$ is pure.

Since there are several simplicial complexes associated to $M$, we will denote this one $I N(M)=(E, \mathcal{I})$. It is often called the independence complex of $M$.

The two most important motivating examples of matroids are the following.

- (Linear Algebra) Let $E$ be a set of vectors in a vector space, and let $\mathcal{I}$ consist of the subsets of $E$ which are linearly independent. Then $(E, \mathcal{I})$ is a linear matroid.

- (Graph Theory) Let $E$ be the set of edges of an undirected graph $G$, and let $\mathcal{I}$ consist of the sets of edges which contain no cycle. Then $(E, \mathcal{I})$ is a graphic matroid.

For any matroid $M=(E, \mathcal{I})$, it is customary to call the sets in $\mathcal{I}$ independent. The facets of a matroid are called bases. The set of all bases is denoted $\mathcal{B}$.

Example 2.2. The simplest example of a matroid is the uniform matroid $U_{k, n}$, whose ground set is $[n]$ and whose independent sets are all the subsets of $[n]$ of cardinality at most $k$. The uniform matroid $U_{1,3}$ is going to play an important role later.

The minimal non-faces of $M$, that is, the minimal dependent sets, are called circuits. The circuits of a matroid have a special structure [19]:

Lemma 2.3 (Circuit Elimination Property). If $\gamma_{1}$ and $\gamma_{2}$ are circuits of a matroid and $c \in \gamma_{1} \cap \gamma_{2}$, then there is a circuit $\gamma_{3}$ that is contained in $\gamma_{1} \cup \gamma_{2}-c$. 
Duality. Matroids have a notion of duality which generalizes orthogonal complements in linear algebra and dual graphs in graph theory.

Let $M$ be a matroid with bases $\mathcal{B}$. Then the set

$$
\mathcal{B}^{*}=\{E-B: B \text { is a basis of } M\}
$$

is the collection of bases of a matroid $M^{*}=\left(E, \mathcal{B}^{*}\right)$, called the dual matroid $M^{*}$. The circuits of the dual matroid $M^{*}$ are called the cocircuits of $M$.

Deletion, contraction, and minors. We say that an element $e \in E$ is a loop of a matroid $M$ if it is contained in no basis; that is, if $\{e\}$ is a dependent set. Dually, $e$ is a coloop if it is contained in every basis of $M$.

The deletion $M \backslash e$ of a non-coloop $e \in E$ is the matroid on $E-e$ whose bases are the bases of $M$ that do not contain $e$. We also call this the restriction of $M$ to $E-e$. Dually, the contraction $M / e$ of a non-loop $e \in E$ is the matroid on $E-e$ whose bases are the subsets $B$ of $E-e$ such that $B \cup e$ is a basis of $M$.

It is easy to see that any sequence of deletions and contractions of different elements commutes. We say that a matroid $M^{\prime}$ is a minor of a matroid $M$ if $M^{\prime}$ is isomorphic to a matroid obtained from $M$ by performing a sequence of deletions and contractions.

Fundamental circuits and cocircuits. Given a basis $B$ and an element $e \in E-B$ there is a unique circuit contained in $B \cup e$, called the fundamental circuit of $e$ with respect to $B$. It is given by

$$
\operatorname{Circ}(B, e)=\{x \in E: B \cup e-x \in \mathcal{B}\} .
$$

Given a basis $B$ and an element $i \in B$ there is a unique cocircuit disjoint with $B-i$, called the fundamental cocircuit of $i$ with respect to $B$. It is given by

$$
\operatorname{Cocirc}(B, i)=\{x \in E: B \cup x-i \in \mathcal{B}\} \text {. }
$$

Note that the cocircuit $\operatorname{Cocirc}(B, i)$ in $M$ equals the $\operatorname{circuit} \operatorname{Circ}(E-B, i)$ in the dual $M^{*}$.

Basis activities. Let $<$ be a linear order on the ground set $E$. For a basis $B$, define the sets:

$$
\begin{aligned}
& E A(B)=\{e \in E-B: \min (\operatorname{Circ}(B, e))=e\} \\
& E P(B)=\{e \in E-B: \min (\operatorname{Circ}(B, e)) \neq e\}
\end{aligned}
$$

The elements of $E A(B)$ and $E P(B)$ are called externally active and externally passive with respect to $B$, respectively. Note that $E A(B) \uplus E P(B)=E-B$, where $\uplus$ denotes a disjoint union.

Dually, let

$$
\begin{aligned}
& I A(B)=\{i \in B: \min (\operatorname{Cocirc}(B, i))=i\} \\
& I P(B)=\{i \in B: \min (\operatorname{Cocirc}(B, i)) \neq i\}
\end{aligned}
$$

The elements of $I A(B)$ and $I P(B)$ are called internally active and internally passive with respect to $B$, respectively. Note that $I A(B) \uplus I P(B)=B$. Also note that the internally 
active/passive elements with respect to basis $B$ in $M$ are the externally active/passive elements with respect to basis $E-B$ in $M^{*}$.

The following elegant result of Tutte [26] (for graphs) and Crapo [7] (for arbitrary matroids) underlies many of the results of [2] and this paper.

Theorem 2.4. [7, Proposition 5.12] Let $M$ be a matroid on the ground set $E$ and let < be a linear order on $E$.

1. Every subset $A$ of $E$ can be uniquely written in the form $A=B \cup X-Y$ for some basis $B$, some subset $X \subseteq E A(B)$, and some subset $Y \subseteq I A(B)$. Equivalently, the intervals $[B-I A(B), B \cup E A(B)]$ form a partition of the poset $2^{E}$ of subsets of $E$ ordered by inclusion.

2. Every independent set $I$ of $E$ can be uniquely written in the form $I=B-Y$ for some basis $B$ and some subset $Y \subseteq I A(B)$. Equivalently, the intervals $[B-I A(B), B]$ form a partition of the independence complex $I N(M)$.

The Tutte polynomial of $M$ is

$$
T_{M}(x, y)=\sum_{B \text { basis }} x^{|I A(B)|} y^{|E A(B)|} .
$$

It follows from the work of Crapo and Tutte $[7,26]$ that this polynomial does not depend on the chosen order $<$. The Tutte polynomial is the most important matroid invariant, because it answers an innumerable amount of questions about the combinatorics, algebra, geometry, and topology of matroids and related objects. For more information, see [5].

The external activity complex. Let $M$ be a matroid on $E$. Let $\bar{E}=\{\bar{e}: e \in E\}$ be a second copy of $E$, and let $[[E]]=E \uplus \bar{E}$. This set of size $2|E|$ will be the ground set of the external activity complex of $M$. For each subset $S \subseteq E$ we write $\bar{S}:=\{\bar{s} \mid s \in S\} \subset \bar{E}$. Therefore, each subset of [[E]] can be written uniquely in the form $S_{1} \cup \overline{S_{2}}$ for $S_{1}, S_{2} \subseteq E$.

Our main object of study is the following.

Theorem 2.1. [2] Let $M=(E, \mathcal{B})$ be a matroid and let $<$ be a linear order on $E$. $M$. There is a simplicial complex called the external activity complex $\operatorname{Act}_{<}(M)$ on ground set $[[E]]$ such that

1. The facets are $F(B):=B \cup E P(B) \cup \overline{B \cup E A(B)}$ for every basis $B \in \mathcal{B}$.

2. The minimal non-faces are $S(\gamma)=c \cup \overline{\gamma-c}$ for every circuit $\gamma$, where $c$ is the $<$-smallest element of $\gamma$.

The complement of the facet $F(B)$ in $[[E]]$ is $G(B)=E A(B) \cup \overline{E P(B)}$.

Las Vergnas's three active orders. Given a matroid $M=(E, \mathcal{B})$ and a total order $<$ on the ground set of $M$, LasVergnas introduced the following three active orders. In each case, he proved that there are several equivalent definitions. 
Definition 2.5. The external order $<_{\text {ext }}$ on $\mathcal{B}$ is characterized by the following equivalent properties for two bases $A$ and $B$ :

1. $A \leqslant$ ext $B$,

2. $A \subseteq B \cup E A(B)$,

3. $A \cup E A(A) \subseteq B \cup E A(B)$,

4. $B$ is the lexicographically largest basis contained in $A \cup B$.

This poset is graded with $r(B)=|E A(B)|$. Adding a minimum element turns it into a lattice.

Definition 2.6. The internal order $<_{\text {int }}$ on $\mathcal{B}$ is characterized by the following equivalent properties for two bases $A$ and $B$ :

1. $A \leqslant$ int $B$,

2. $A-I A(A) \subseteq B$,

3. $A-I A(A) \subseteq B-I A(B)$,

4. $A$ is the lexicographically smallest basis containing $A \cup B$.

This poset is graded with $r(B)=r-|I A(B)|$. Adding a maximum element turns it into a lattice.

The internal and external orders are consistent in the sense that $A \leqslant_{i n t} B$ and $B \leqslant$ ext $A$ imply $A=B$. Therefore the following definition makes sense.

Definition 2.7. The external/internal order $<_{\text {ext } / \text { int }}$ is the weakest order which simultaneously extends the external and the internal order. It is characterized by the following equivalent properties for two bases $A$ and $B$ :

1. $A \leqslant$ ext/int $B$,

2. $I P(A) \cap E P(B)=\varnothing$,

This poset is a lattice. It is not necessarily graded.

Note that Theorem 2.5.4 and 2.6.4 imply the following.

Proposition 2.8. The lexicographic order $<_{\text {lex }}$ on $\mathcal{B}$ is a linear extension of the three posets $<_{i n t},<_{e x t}$, and $<_{e x t / i n t}$. In symbols, any of $A<_{i n t} B, A<_{e x t} B$ or $A<_{e x t / i n t} B$ implies $A<_{\text {lex }} B$.

\subsection{Shellability and the $h$-vector}

Shellability. Shellability is a combinatorial condition on a simplicial complex that allows us to describe its topology easily. A simplicial complex is shellable if it can be built up by introducing one facet at a time, so that whenever we introduce a new facet, its intersection with the previous ones is pure of codimension 1. More precisely:

Definition 2.9. Let $\Delta$ be a pure simplicial complex. A shelling order is an order of the facets $F_{1}, \ldots F_{k}$ such for every $i<j$ there exist $k<j$ and $f \in F_{j}$ such that $F_{i} \cap F_{j} \subseteq$ $F_{k} \cap F_{j}=F_{j}-f$. If a shelling order exists, then we call $\Delta$ shellable.

Given a shelling order and a facet $F_{j}$, there is a subset $\mathcal{R}\left(F_{j}\right)$ such that for every $A \subseteq F_{j}$, we have $A \nsubseteq F_{i}$ for all $i<j$ if and only if $\mathcal{R}\left(F_{j}\right) \subseteq A$. Equivalently, when we add facet $F_{j}$ to the complex, the new faces that we introduce are precisely those in the interval $\left[\mathcal{R}\left(F_{j}\right), F_{j}\right]$. The set $\mathcal{R}\left(F_{j}\right)$ is called the restriction set of $F_{j}$ in the shelling. 
The $\boldsymbol{f}$-vector and $\boldsymbol{h}$-vector. The $f$-vector of a $(d-1)$-dimensional simplicial complex $\Delta$ is $\left(f_{0}, \ldots, f_{d}\right)$ where $f_{i}$ is the number of faces of $\Delta$ of size $i$. The h-vector $\left(h_{0}, \ldots, h_{d}\right)$ is an equivalent way of storing this information; it is defined by the relation

$$
f_{0}(x-1)^{d}+f_{1}(x-1)^{d-1}+\cdots+f_{d}(x-1)^{0}=h_{0} x^{d}+h_{1} x^{d-1}+\cdots+h_{d} x^{0} .
$$

This polynomial is also known as the shelling polynomial $h_{\Delta}(x)$, due to the following description of the $h$-vector for shellable complexes.

Proposition 2.10. [4, Proposition 7.2.3] If $F_{1}, \ldots, F_{k}$ is a shelling order for a $(d-1)$ dimensional simplicial complex $\Delta$, then

$$
h_{i}:=\left|\left\{j:\left|\mathcal{R}\left(F_{j}\right)\right|=i\right\}\right| .
$$

Note that it is not clear a priori that these numbers should be the same for any shelling order.

Understanding the topology of a shellable simplicial complex is easy once we know the last entry of the $h$-vector, thanks to the following result.

Theorem 2.11. [13, Theorem 12.2(2)] Any geometric realization of a $(d-1)$-dimensional shellable simplicial complex $\Delta$ is homotopy equivalent to a wedge of $h_{d}$ spheres of dimension $d-1$. In particular, if $h_{d}=0$, then every geometric realization of $\Delta$ is contractible.

An important property for matroids is their shellability:

Theorem 2.12. [4, Theorem 7.3.3] The lexicographic order $<_{l e x}$ on the bases of a matroid $M$ gives a shelling order of the independence complex $I N(M)$. Furthermore, the restriction set of a basis $B$ in this shelling order is given by $\operatorname{IP}(B)$.

A straightforward consequence of the previous theorem is that the internal order poset is equal to the poset of bases of $M$ where the order is given by inclusion of restriction sets of the lexicographic shelling order.

\section{Example}

Before proving our theorems, we illustrate them in an example. Consider the graphic matroid given by the graph of Figure 1. Its bases are all the 3 -subsets of [5] except $\{1,2,3\}$ and $\{1,4,5\}$. Under the standard order $1<2<3<4<5$ on the ground set, Table 1 records the basis activity of the various bases.

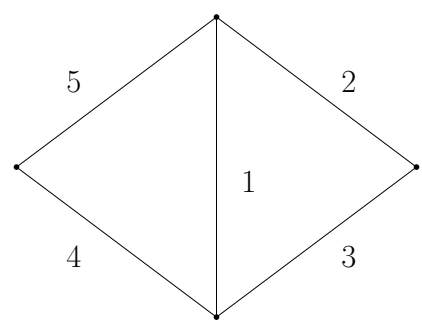

Figure 1: A graphic matroid. 


\begin{tabular}{|c||c|c|c|c|}
\hline$B$ & $E P(B)$ & $E A(B)$ & $I P(B)$ & $I A(B)$ \\
\hline 124 & 35 & $\varnothing$ & $\varnothing$ & 124 \\
\hline 125 & 45 & $\varnothing$ & 5 & 12 \\
\hline 134 & 25 & $\varnothing$ & 3 & 14 \\
\hline 135 & 24 & $\varnothing$ & 35 & 1 \\
\hline 234 & 5 & 1 & 23 & 4 \\
\hline 235 & 4 & 1 & 235 & $\varnothing$ \\
\hline 245 & 3 & 1 & 45 & 2 \\
\hline 345 & $\varnothing$ & 12 & 345 & $\varnothing$ \\
\hline
\end{tabular}

Table 1: The bases $B$ together with their sets of externally passive, externally active, internally passive, and internally active elements.

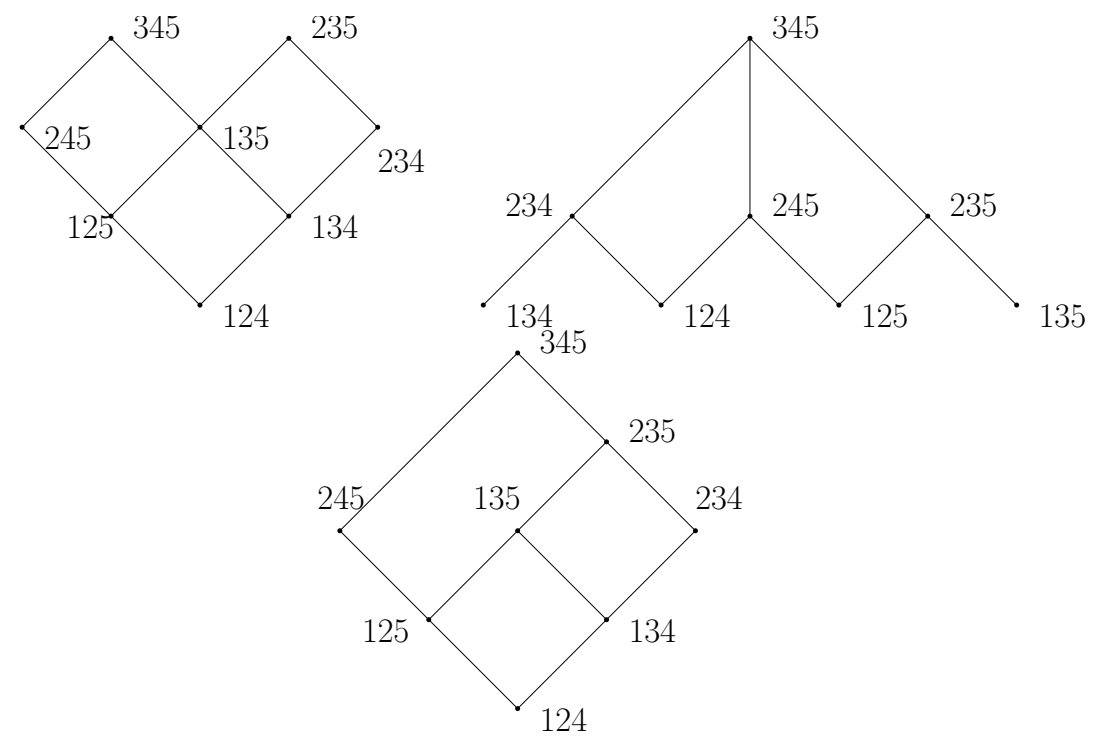

Figure 2: The active orders $<_{i n t},<_{e x t}$, and $<_{\text {ext } / \text { int }}$, respectively.

The resulting internal, external, and external/internal orders $<_{\text {int }},<_{\text {ext }},<_{\text {ext } / \text { int }}$ are shown in Figure 2. By Theorems 2.5, 2.6, and 2.7, these three orders are isomorphic to the three families of sets $\{B \cup E A(B): B$ basis $\},\{B-I A(B): B$ basis $\}$, and $\{B \cup E A(B)-I A(B): B$ basis $\}$, partially ordered by containment.

Table 1 lists the bases in lexicographic order $<_{\text {lex }}$, and this is a shelling order for the independence complex $I N(M)$ by Theorem 2.12. The restriction set for each basis $B$ is $\mathcal{R}(B)=I P(B)$. For example, when we add facet 134 in the third step of the shelling, this means that the new faces that appear are the four sets in the interval $[\mathcal{R}(134), 134]=[3,134] ;$ that is, faces $3,13,34$, and 134 .

Our goal is to shell the external activity complex $\operatorname{Act}_{<}(M)$ whose facets, listed in Table 2, are the sets $F(B)=B \cup E P(B) \cup \overline{B \cup E A(B)}$. Since $\overline{1}, 3,4$, and 5 are in all facets of $\operatorname{Act}_{<}(M)$, we remove them, and shell the resulting reduced external activity complex $A c t_{<}^{\bullet}(M)$. Our main result, Theorem 1.2, states that any linear extension of the external/internal order $<_{\text {ext }}$ int gives a shelling order for this complex. For example, we 
may again consider the lexicographic order, which is indeed a linear extension of $<_{\text {ext }}$ int .

\begin{tabular}{|c|c|c|c|}
\hline$B$ & $F(B)$ & $F(B)^{\bullet}$ & $\mathcal{R}(F(B))$ \\
\hline 124 & $12345 \overline{124}$ & $12 \overline{24}$ & $\varnothing$ \\
125 & $12345 \overline{125}$ & $12 \overline{25}$ & $\overline{5}$ \\
134 & $12345 \overline{134}$ & $12 \overline{34}$ & $\overline{3}$ \\
135 & $12345 \overline{135}$ & $12 \overline{35}$ & $\overline{35}$ \\
234 & $2345 \overline{1234}$ & $2 \overline{234}$ & $\overline{23}$ \\
235 & $2345 \overline{1235}$ & $2 \overline{235}$ & $\overline{235}$ \\
245 & $2345 \overline{1245}$ & $2 \overline{245}$ & $\overline{45}$ \\
345 & $345 \overline{12345}$ & $\overline{2345}$ & $\overline{345}$ \\
\hline
\end{tabular}

Table 2: The bases $B$ of $M$, the corresponding facets $F(B)$ and $F(B)^{\bullet}$ of $\operatorname{Act}_{<}(M)$ and $\operatorname{Act}_{<}^{\bullet}(M)$, and their (shared) restriction set $\mathcal{R}(F(B))$ in the shelling.

For each basis $B$, Table 2 lists the corresponding facet $F(B)$ of $\operatorname{Act}_{<}(M)$, the corresponding facet $F(B)^{\bullet}$ of $\operatorname{Act}_{<}^{\bullet}(M)$, and the restriction set of the facet $F(B)$ in the shelling. This restriction set is $\mathcal{R}(F(B))=\overline{I P(B)}$. For example, when we add facet $12 \overline{34}$ to the complex $\operatorname{Act}_{<}^{\bullet}(M)$ in the third step of the shelling, the new faces that appear are the eight sets in the interval $[\mathcal{R}(12 \overline{34}), 12 \overline{34}]=[\overline{3}, 12 \overline{34}]$.

Notice that we can embed $I N(M) \longrightarrow \operatorname{Act}_{<}^{\bullet}(M)$ by sending $1 \rightarrow 1,2 \rightarrow \overline{2}, 3 \rightarrow \overline{3}, 4 \rightarrow$ $\overline{4}, 5 \rightarrow \overline{5}$. The latter complex has the same $h$-vector and is contractible. Therefore, it is no coincidence that the shellings of $I N(M)$ and $A_{c} t_{<}(M)$ are related. In fact, we will prove that any shelling order for $\operatorname{Act}_{<}(M)$ is a shelling order for $I N(M)$. Theorem 1.2 then gives:

any linear extension of $<_{\text {ext } / \text { int }}$ is a shelling order for $I N(M)$ and $\operatorname{Act}_{<}(M)$.

We conclude this section with two examples showing that the linear extensions of the internal and external orders $<_{i n t}$ and of $<_{e x t}$ are not necessarily shelling orders for $\operatorname{Act}_{<}(M)$.

Example 3.1. Consider any linear extension of $<_{e x t}$ starting with 124 and 135 in that order, such as:

$$
124,135,125,134,234,235,245,345 .
$$

This is not a shelling order for $I N(M)$ because the second facet 135 intersects the first facet 124 in codimension 2. By Corollary 4.3 (or directly by inspection), this is not a shelling order for $\operatorname{Act}_{<}(M)$ either. Therefore:

a linear extension of $<_{\text {ext }}$ need not be a shelling order for $I N(M)$ or for $\operatorname{Act}_{<}(M)$.

Example 3.2. Consider the following linear extension of $<_{i n t}$ :

$$
124,125,134,135,245,345,234,235
$$

which gives the following order on the facets:

$$
12 \overline{24}, 12 \overline{25}, 12 \overline{34}, 12 \overline{35}, 2 \overline{245}, \overline{2345}, 2 \overline{234}, 2 \overline{235}
$$


This is a shelling of $I N(M)$ by Theorem 1.3. However, it is not a shelling of $\operatorname{Act}_{<}(M)$ and $\operatorname{Act}_{<}^{\bullet}(M)$. To see this, suppose we introduce the facets of $\operatorname{Act}_{<}^{\bullet}(M)$ in the order above. When we introduce the sixth facet $\overline{2345}$ we introduce two new minimal faces: $\overline{23}$ and $\overline{345}$; so this is not a shelling order for $\operatorname{Act}_{<}(M)$. Hence

a linear extension of $<_{i n t}$ is a shelling order for $I N(M)$, but not necessarily for $\operatorname{Act}_{<}(M)$.

In summary, combining (1), (2), and (3), we see that the hypotheses of Theorems 1.2 and 1.3 are as strong as possible in the context of LasVergnas's active orders.

\section{Shellability of the external activity complex}

In this section we prove our main result, which states that the external activity complex is shellable. We begin by proving two technical lemmas.

Lemma 4.1. Let $M$ be a matroid on an ordered ground set, and let $A, C$ be bases of $M$. There exist $c \in E P(A) \cap C$ and $a<c$ such that $C-c \cup a$ is a basis if and only if $A \nsupseteq$ ext/int $C$ in LasVergnas's external/internal order.

Proof. Given $c \in C$, we can find an element $a<c$ with $C-c \cup a \in \mathcal{B}$ if and only if $c \in$ $I P(C)$. To find such an element $c$ with the additional condition that $c \in E P(A)$, we need $I P(C) \cap E P(A) \neq \varnothing$; this is equivalent to $A \nsupseteq$ ext/int $C$ in LasVergnas's external/internal order by Theorem 2.7.2.

A total order $<$ on the set $\mathcal{B}$ of bases of $M$ induces an order on the set of facets $\{F(B): B \in \mathcal{B}\}$ of the external activity complex $\operatorname{Act}_{<}(M)$. We now characterize the shelling orders on $\operatorname{Act}_{<}(M)$.

Lemma 4.2. Let $\mathcal{B}$ be the set of bases of a matroid $M$. A total order $<$ on the set $\mathcal{B}$ induces a shelling of the external activity complex $\operatorname{Act}_{<}(M)$ if and only if for any bases $A<C$ there exists a basis $B<C$ such that

(a) $B=X \cup b$ and $C=X \cup c$ for some $b \neq c$.

(b) $c \notin A$ and $c \in E A(B)$ if and only if $c \in E A(A)$.

(c) For any $d \notin B \cup C=X \cup b \cup c$ we have $d \in E A(B)$ if and only if $d \in E A(C)$

Proof. By definition, $<$ induces a shelling order if for every $A<C$ there exist $B<C$ and $c^{ \pm} \in F(C)$ (where $c^{ \pm}$equals $c$ or $\bar{c}$ for some $c \in E$ ) such that

$$
F(A) \cap F(C) \subset F(B) \cap F(C)=F(C)-c^{ \pm} .
$$

Recalling that $G(D)=E A(D) \cup \overline{E P(D)}$ is the complement of $F(D)$ in $[[E]]$ for each basis $D$, this is equivalent to

$$
G(A) \cup G(C) \supset G(B) \cup G(C)=G(C) \cup c^{ \pm} .
$$


Define the support of $S \subset[[E]]$ to be $\operatorname{supp}(S)=\{i \in E: i \in S$ or $\bar{i} \in S\}$. Notice that we have $\operatorname{supp}(G(D))=E-D$ for any basis $D$. Then

$$
|E|-|B \cap C|=|\operatorname{supp}(G(B) \cup G(C))|=\left|\operatorname{supp}\left(G(C) \cup c^{ \pm}\right)\right|=|E|-r+1 .
$$

where $r$ is the rank of the matroid. This implies (a).

If (c) was not satisfied for some $d \notin B \cup C$, we would find both $d$ and $\bar{d}$ in $G(B) \cup$ $G(C)=G(C) \cup c$, a contradiction. Finally, $c^{ \pm}$is in $G(A)$ and $G(B)$, which implies (b).

The converse follows by a very similar argument.

Corollary 4.3. If a total order $<$ on $\mathcal{B}$ induces a shelling of the external activity complex $\operatorname{Act}_{<}(M)$, then it also induces a shelling of the independence complex $I N(M)$.

Proof. Let $A<C$ and assume that $B<C$ satisfy conditions (a), (b), and (c) of Lemma 4.2. Since $\operatorname{supp}(G(D))=E-D$ for every basis $D$, the containment $G(A) \cup G(C) \supset$ $G(B) \cup G(C)$ gives $E-(A \cap C) \supset E-(B \cap C)$, which implies $A \cap C \subset B \cap C=X=C-c$. Hence the total order $<$ induces a shelling order of $I N(M)$.

Now we are ready to prove our main theorem.

Theorem 1.2. Let $M=(E, \mathcal{B})$ be a matroid, and let $<$ be a linear order on the ground set $E$. Any linear extension of LasVergnas's external/internal order $<_{\text {ext } / \text { int }}$ of $\mathcal{B}$ induces a shelling of the external activity complex $\operatorname{Act}_{<}(M)$.

Proof. We use the characterization of Lemma 4.2. Consider bases $A<C$; we will find the desired basis in two steps. We construct a basis $B$ and, if necessary, a second basis $B^{\prime}$, and we will show that one of them satisfies the conditions (a),(b), (c) of Lemma 4.2.

Step 1. Since $A \Varangle_{\text {ext } / \text { int }} C$, we first use Lemma 4.1 to find $c \in E P(A) \cap C$ and a minimal element $b<c$ such that

$$
B=X \cup b
$$

is a basis, where $X=C-c$. The minimality of $b$ implies that $b$ is minimum in $\operatorname{Cocirc}(B, b)$, so $b \in I A(B)$. Therefore $B \backslash I A(B) \subseteq X \subseteq C$. Theorem 2.6 then implies that $B<_{\text {int }} C$, which in turn gives $B<_{\text {ext } / \text { int }} C$, and hence $B<C$.

Property (a) is clearly satisfied. By construction $c \notin A$ and $c \in E P(A)$. Since $b<c$ is in $\operatorname{Circ}(B, c)$, we have $c \in E P(B)$. Therefore (b) is also satisfied. Property (c) does not always hold; let us analyze how it can fail, and adjust $B$ accordingly if necessary.

Suppose (c) fails for an element $d \notin B \cup C$; call such an element a $\{B, C\}$ external disagreement. This means that $d$ is minimum in one of the fundamental circuits $\beta=$ $\operatorname{Circ}(B, d)$ and $\gamma=\operatorname{Circ}(C, d)$ but not in the other one.

Since they have different minima, we have $\beta \neq \gamma$; so using circuit elimination, we can find a circuit $\alpha \subseteq \beta \cup \gamma-d$. This circuit must contain $b$ and $c$, or else it would be contained in basis $B$ or $C$. This implies that

$$
b, c \in \alpha, \quad b, d \in \beta, \quad c, d \in \gamma .
$$

It follows that $D=X \cup d=(B \cup d)-b$ is a basis. By the uniqueness of fundamental circuits, we must have

$$
\begin{aligned}
& \alpha=\operatorname{Circ}(B, c)=\operatorname{Circ}(C, b), \quad \beta=\operatorname{Circ}(B, d)=\operatorname{Circ}(D, b), \\
& \gamma=\operatorname{Circ}(C, d)=\operatorname{Circ}(D, c) .
\end{aligned}
$$

Taking into account that $b<c$, we consider three cases: 


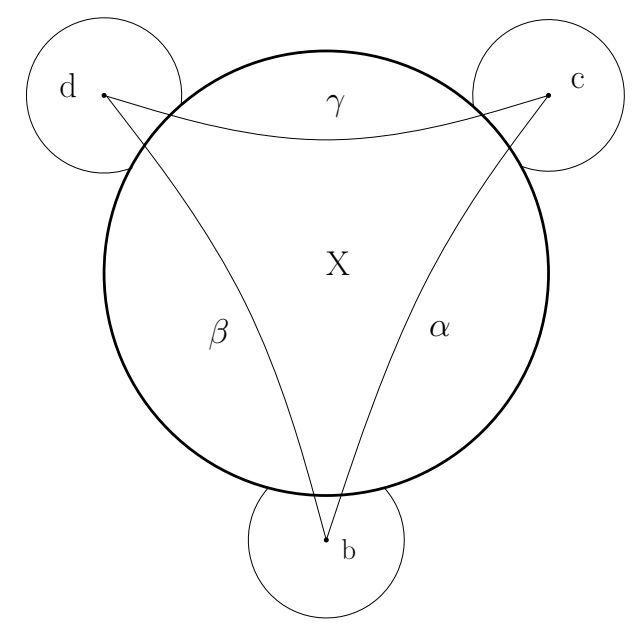

Figure 3: The bases $B=X \cup b, C=X \cup c$, and $D=X \cup d$ and the fundamental circuits $\beta, \gamma, \alpha$.

- 1. $\boldsymbol{b}<\boldsymbol{c}<\boldsymbol{d}$ : Since $b \in \operatorname{Circ}(B, d)=\beta$ and $c \in \operatorname{Circ}(C, d)=\gamma, d$ is minimum in neither $\beta$ nor $\gamma$, a contradiction.

- 2. $\boldsymbol{d}<\boldsymbol{b}<\boldsymbol{c}$ : The minimality of $b$ implies that $X \cup d=D$ is not a basis, a contradiction.

- 3. $\boldsymbol{b}<\boldsymbol{d}<\boldsymbol{c}$ : Since $d$ is not minimum in $\operatorname{Circ}(B, d)=\beta \ni b$, we have $d \in E P(B)$; so $d$ is a $\{B, C\}$ external disagreement if and only if $d \in E A(C)$.

We conclude that, under the above hypotheses,

$d$ is a $\{B, C\}$ external disagreement $\Longleftrightarrow X \cup d$ is a basis, $b<d<c$, and $d \in E A(C)$.

If there are no $\{B, C\}$ external disagreements, $B$ is our desired basis. Otherwise, proceed as follows.

Step 2. Define the basis

$$
B^{\prime}=X \cup b^{\prime}
$$

where $b^{\prime}$ is the largest $\{B, C\}$ external disagreement. We have $b<b^{\prime}<c$ and $b^{\prime} \in E A(C)$. It follows that $B^{\prime} \subset C \cup E A(C)$, so $B^{\prime}<_{e x t} C$ by Theorem 2.5. This implies that $B^{\prime}<_{\text {ext } / \text { int }} C$, which in turn gives $B^{\prime}<C$. Now we claim that $B^{\prime}$ satisfies conditions (a),(b),(c) of Lemma 4.2 .

Property (a) is clearly satisfied. By construction $c \notin A$ and $c \in E P(A)$. Since $b^{\prime}<c$ is in $\operatorname{Circ}\left(B^{\prime}, c\right)$, we have $c \in E P\left(B^{\prime}\right)$, so (b) holds. To show (c), assume contrariwise that $d^{\prime} \notin X \cup b^{\prime} \cup c$ is a $\left\{B^{\prime}, C\right\}$ external disagreement; that is, it is minimum in one of the fundamental circuits $\beta^{\prime}=\operatorname{Circ}\left(B^{\prime}, d^{\prime}\right)$ and $\gamma^{\prime}=\operatorname{Circ}\left(C, d^{\prime}\right)$ but not in the other.

As in Step $1, D^{\prime}=X \cup d^{\prime}$ must be a basis, and we have circuits

$$
\begin{aligned}
\alpha^{\prime} & =\operatorname{Circ}\left(B^{\prime}, c\right)=\operatorname{Circ}\left(C, b^{\prime}\right), \quad \beta^{\prime}=\operatorname{Circ}\left(B^{\prime}, d^{\prime}\right)=\operatorname{Circ}\left(D^{\prime}, b^{\prime}\right), \\
\gamma^{\prime} & =\operatorname{Circ}\left(C, d^{\prime}\right)=\operatorname{Circ}\left(D^{\prime}, c\right) .
\end{aligned}
$$

with $b^{\prime}, c \in \alpha^{\prime}, b^{\prime}, d^{\prime} \in \beta^{\prime}, c, d^{\prime} \in \gamma^{\prime}$.

Once again, in view of $b^{\prime}<c$, we consider three cases: 


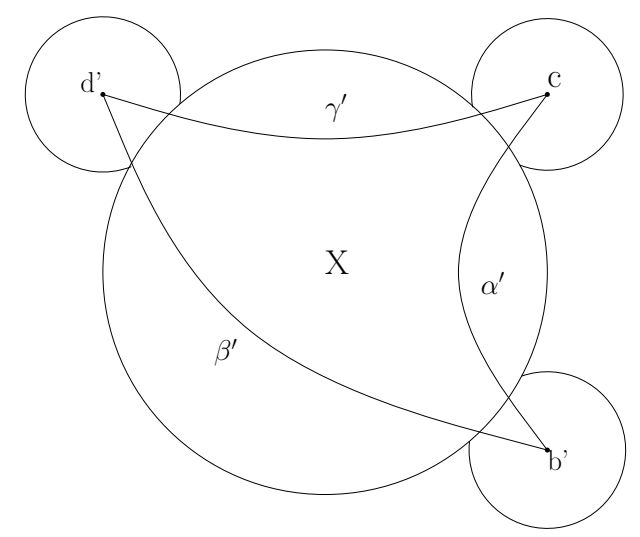

Figure 4: The bases $B^{\prime}=X \cup b^{\prime}, C=X \cup c$, and $D^{\prime}=X \cup d^{\prime}$ and the fundamental circuits $\beta^{\prime}, \gamma^{\prime}, \alpha^{\prime}$.

- Case $1 \boldsymbol{b}^{\prime}<\boldsymbol{c}<\boldsymbol{d}^{\prime}$ : Since $b^{\prime} \in \operatorname{Circ}\left(B, d^{\prime}\right)=\beta^{\prime}$ and $c \in \operatorname{Circ}\left(C, d^{\prime}\right)=\gamma, d^{\prime}$ is minimum in neither $\beta$ nor $\gamma$, a contradiction.

- Case $2 \boldsymbol{d}^{\prime}<\boldsymbol{b}^{\prime}<\boldsymbol{c}$ : If $d^{\prime} \in E A\left(B^{\prime}\right)$ then $d^{\prime}=\min \beta^{\prime}$. Since $b^{\prime} \in E A(C)$, we have $b^{\prime}=\min \alpha^{\prime}$. Because they have different minima, we have $\beta^{\prime} \neq \alpha^{\prime}$, so we can use circuit elimination to find a circuit $\gamma^{\prime \prime} \subseteq\left(\alpha^{\prime} \cup \beta^{\prime}\right)-b^{\prime}$. Again, that circuit must contain $c$ and $d^{\prime}$ or else it would be contained in $C$ or $D^{\prime}$. Therefore, by the uniqueness of fundamental circuits, $\gamma^{\prime \prime}=\gamma^{\prime}$. Now, since $\gamma^{\prime} \subseteq\left(\alpha^{\prime} \cup \beta^{\prime}\right)-b^{\prime}$ and $d^{\prime} \in \gamma^{\prime}$, we have $d^{\prime}=\min \gamma^{\prime}$ and $d^{\prime} \in E A(C)$.

Similarly, if $d^{\prime} \in E A(C)$ then $d^{\prime}=\min \gamma^{\prime}$. Since $b^{\prime} \in E A(C)$, we have $b^{\prime}=\min \alpha^{\prime}$. As above, we can conlcude that $\beta^{\prime} \subseteq\left(\alpha^{\prime} \cup \gamma^{\prime}\right)-c$ and $d^{\prime} \in \beta^{\prime}$, we have $d^{\prime}=\min \beta^{\prime}$ and $d^{\prime} \in E A\left(B^{\prime}\right)$.

In either case, we get a contradiction.

- Case $3 \boldsymbol{b}^{\prime}<\boldsymbol{d}^{\prime}<\boldsymbol{c}$ Since $d^{\prime}$ is not minimum in $\beta^{\prime}=\operatorname{Circ}\left(B, d^{\prime}\right) \ni b^{\prime}$, if $d^{\prime}$ is a $\left\{B^{\prime}, C\right\}$ external disagreement, it must be minimum in $\gamma=\operatorname{Circ}\left(C, d^{\prime}\right)$; that is, $d^{\prime} \in E A(C)$. We have $b<b^{\prime}<d^{\prime}<c$, and $X \cup d^{\prime}$ is a basis. Therefore, recalling (4), $d^{\prime}$ is also a $\{B, C\}$ external disagreement, contradicting the maximality of $b^{\prime}$.

In conclusion, there are no $\left\{B^{\prime}, C\right\}$ external disagreements, and property (c) holds. Therefore the basis $B^{\prime}$ has all required properties.

Corollary 4.4. Any linear extension of the external/internal order $<_{\text {ext } / \text { int }}$ gives a shelling order for the independence complex $I N(M)$.

Proof. This follows from Theorem 1.2 and Corollary 4.3.

In fact, we now prove a stronger result. We begin with a useful lemma.

Lemma 4.5. Let $I$ be an independent set of $M$ and let $C$ be any basis that contains $I$. If $B$ is the lexicographically smallest basis that contains $I$ then $B \leqslant_{i n t} C$.

Proof. By Theorem 2.6.4, we need to show that $B$ is the lexicographically smallest basis that contains $B \cap C$. To do so, assume there is a basis $A \supseteq B \cap C$ with $A<_{\text {lex }} B$ Then $A \supseteq B \cap C \supseteq I$, contradicting the minimality of $B$. 
Theorem 1.3. Any linear extension of the internal order $<_{\text {int }}$ gives a shelling order of the independence complex $I N(M)$.

Proof. Let $<$ be any linear extension of $<_{\text {int }}$, and let $A<C$ be bases, so $A \ngtr_{\text {int }} C$. We claim that there exists $B<_{i n t} C$ (and hence $B<C$ ) such that $A \cap C \subseteq B \cap C=C-c$ for some $c$ in $C$. This will prove the desired result.

To show this, let $D$ be the lexicographically smallest basis that contains $A \cap C$. Notice that $D \neq C$ because $A \ngtr_{\text {int }} C$, using Theorem 2.6.4. Let $d$ be smallest element in $D-C$ and let $c$ be any element of $C-D$ such that $C^{\prime}=C-c \cup d$ is a basis. Also notice that $D<_{\text {int }} C$ by Lemma 4.5; and since $<_{\text {lex }}$ is a linear extension of $<_{\text {int }}$, we have $D<_{\text {lex }} C$. This gives $d=\min (D-C)<\min (C-D) \leqslant c$, and therefore $C^{\prime}<_{\text {lex }} C$.

Put $X=C-c$ and let $B$ be the lexicographically smallest basis that contains $X$. Since $C^{\prime}$ contains $X, B \leqslant_{l e x} C^{\prime}<_{\text {lex }} C$, so $B \neq C$. Therefore $B<_{\text {int }} C$ by Lemma 4.5. Also note that, since $c \notin D \supset A \cap C$ and $c \in C$, we must have $c \notin A$. This gives $A \cap C \subseteq C-c=X$, and therefore $A \cap C \subseteq B \cap C=X$. It follows that $B$ satisfies the desired properties.

\section{The $h$-vector}

We now describe the restriction sets for the shellings of Theorem 1.2.

Proposition 5.1. Let $<$ be any linear extension of $<_{\text {ext } / \text { int }}$, and regard it as a shelling order for $I N(M)$. Then the restriction set of each facet $C$ (which is a basis of $M$ ) is $I P(C)$.

Proof. We need to show $I P(C)$ is the minimum subset of $C$ which is not a subset of a basis $B<C$.

To show that $I P(C)$ indeed has this property, assume that if $I P(C) \subseteq B$. Then by Theorem 2.6.2, we have $C \leqslant{ }_{\text {int }} B$ and hence $C \leqslant B$, as desired.

To show minimality, let $U \subsetneq I P(C)$. By Theorem 2.4.2 we can find a basis $A$ such that $A-I A(A) \subseteq U \subseteq A$. This gives $A-I A(A) \subseteq U \subsetneq C-I A(C)$, which in light of Theorem 2.6.3 gives $A<_{i n t} C$, and hence $A<C$. Therefore $U$ is a subset of $A$ with $A<C$, as desired.

Proposition 5.2. Let $<$ be any linear extension of $<_{\text {ext } / \text { int }}$, and regard it as a shelling order for $\operatorname{Act}_{<}(M)$. Then the restriction set of each facet $F(C)$ (where $C$ is a basis of $M)$ is $\overline{I P(C)}$.

Proof. We need to show $\overline{I P(C)}$ is the minimum subset of $F(C)$ which is not a subset of $F(B)$ for any basis $B<C$.

To show $\overline{I P(C)}$ does have this property, assume that $\overline{I P(C)} \subseteq F(B)=B \cup E P(B) \cup$ $\overline{B \cup E A(B)}$ for some basis $B$. Then $I P(C) \subset B \cup E A(B)$, so $I P(C) \cap E P(B)=\varnothing$. By Theorem 2.7.2, $C<_{\text {ext } / \text { int }} B$ so $C<B$, as desired.

To show minimality, let $\bar{U} \subsetneq \overline{I P(C)}$, so $U \subsetneq I P(C)$. By Proposition 5.1, $U$ is contained in a basis $A<C$, and hence $\bar{U}$ is contained in $F(A)$ for that basis, as desired.

As an immediate consequence, we obtain our main enumerative result. 
Theorem 1.4. The $h$-vector of $\operatorname{Act}_{<}(M)$ equals the $h$-vector of $M$.

Proof. This follows from the previous two results, in light of Proposition 2.10.

\section{Topology}

The external activity complex $\operatorname{Act}_{<}(M)$ is a cone; for example, it is easy to see that every facet contains $\overline{\min E}$ and $\max E$. Therefore $\operatorname{Act}_{<}(M)$ is trivially contractible. It is more interesting to study the topology of the reduced external activity complex $\operatorname{Act}_{<}^{\bullet}(M)$, obtained by removing all cone points of $\operatorname{Act}_{<}(M)$. It turns out that Corollary 1.4 gives us enough information to describe it. First we need a few technical lemmas.

Definition 6.1. Define a loop of a simplicial complex $\Delta$ to be an element $l$ of the ground set such that $\{l\}$ is not a face of $\Delta$.

Definition 6.2. An element $e$ of a matroid $M$ is absolutely externally active if it is externally active with respect to every basis not containing it, or absolutely externally passive if it is externally passive with respect to every basis not containing it.

Let $A E A(M)$ and $A E P(M)$ be the respective sets of elements, and call the elements of $A E(M)=A E A(M) \cup A E P(M)$ externally absolute.

Lemma 6.3. The set of cone points of $A c t_{<}(M)$ is $A E P(M) \cup \overline{A E A(M)}$. The ground set of $\operatorname{Act}_{<}^{\bullet}(M)$ is $\{e: e \notin A E P(M)\} \cup\{\bar{e}: e \notin A E A(M)\}$, and this simplicial complex has no loops.

Proof. The first two statements are clear from the definitions. For the last one, if $e \notin$ $A E P(M)$, then we can find a basis $B$ with respect to which $e$ is externally active, so $\{\bar{e}\} \subset F(B)$ is a face of $\operatorname{Act}_{<}^{\bullet}(M)$. Similarly, if $e \notin A E A(M)$, then we can find a basis $B$ with respect to which $e$ is externally passive, so $\{e\} \subset F(B)$ is a face of $\operatorname{Act}_{<}^{\bullet}(M)$.

Lemma 6.4. Let $M=(E, \mathcal{B})$ be a matroid. Every element $e \in E$ is externally absolute if and only if the circuits of $M$ are pairwise disjoint.

Proof. The backward direction is a straightforward consequence of the definitions. To prove the forward direction, we proceed by contradiction. Assume that every element of $M$ is externally absolute, and that we have two circuits $\gamma_{1}$ and $\gamma_{2}$ with $\gamma_{1} \cap \gamma_{2} \neq \varnothing$ whose minimal elements are $c_{1}$ and $c_{2}$, respectively. Consider two cases.

1. If $c_{1}=c_{2}$ then perform circuit elimination to get $\gamma_{3} \subset \gamma_{1} \cup \gamma_{2}-c_{1}$. Let $c_{3}$ be the minimal element of $\gamma_{3}$; without loss of generality assume $c_{3} \in \gamma_{1}$. Then $c_{3}$ is externally active for some basis, as testified by $\gamma_{3}$, and it is externally passive for another basis, as testified by $\gamma_{1}$. Hence $c_{3}$ is not absolute, a contradiction

2. If $c_{1} \neq c_{2}$ and $c \in \gamma_{1} \cap \gamma_{2}$, then perform circuit elimination with $c$ to get a circuit $\gamma_{3} \subset \gamma_{1} \cup \gamma_{2}-c$. Let $c_{3}$ be the minimal element of $\gamma_{3}$; assume $c_{3} \in \gamma_{1}$. If $c_{3}=c_{1}$, then case 1 applies to circuits $\gamma_{1}$ and $\gamma_{3}$, and we get a contradiction. Otherwise, we must have $c_{1}<c_{3}$ since $c_{1}=\min \gamma_{1}$. Therefore $c_{3}$ is externally active for some basis, as testified by $\gamma_{3}$, and externally passive for another basis, as testified by $\gamma_{1}$, a contradiction. 
Proposition 6.5. If a matroid is the disjoint union of circuits, then $\operatorname{Act}_{<}^{\bullet}(M) \cong I N(M)$. Otherwise, $\operatorname{Act}_{<}^{\bullet}(M)$ has a proper subcomplex which is isomorphic to $I N(M)$. The embedding may be chosen so that the image of facet $B$ of $I N(M)$ is a subset of the facet $F(B)$ of $\operatorname{Act}_{<}^{\bullet}(M)$.

Proof. For every $e \in E$ let $e^{\prime}=e$ if $e$ is absolutely externally active, and $e^{\prime}=\bar{e}$ otherwise. The set $E^{\prime}=\left\{e^{\prime}: e \in E\right\}$ is a subset of the vertices of $\operatorname{Act}_{<}^{\bullet}(M)$ by Lemma 6.3. For every basis $B$ of $M$ the set $B^{\prime}=\left\{b^{\prime}: b \in B\right\}$ is a subset of $F(B)$, and hence a face of $\operatorname{Act}_{<}^{\bullet}(M)$. This gives the desired embedding of $I N(M)$ in $\operatorname{Act}_{<}^{\bullet}(M)$.

If $M$ is the disjoint union of circuits, then $E^{\prime}$ equals the ground set of $\operatorname{Act}_{<}^{\bullet}(M)$, and $B^{\prime}$ equals $F(B) \cap E^{\prime}$ for all bases $B$, so this embedding is actually an isomorphism.

If $M$ is not the disjoint union of circuits, by Lemma $6.3, E^{\prime}$ is a proper subset of the ground set of $\operatorname{Act}_{<}^{\bullet}(M)$, so the embedding of $I N(M)$ is a proper subcomplex of $\operatorname{Act}_{<}^{\bullet}(M)$.

Lemma 6.6. If a matroid $M$ of rank $r$ is the disjoint union of circuits, then the independence complex $I N(M)$ is homeomorphic to an $(r-1)$-sphere.

Proof. If $M$ is a single circuit (necessarily of size $r+1$ ), then $I N(M)$ is the boundary of an $r$-simplex, and hence an $(r-1)$-sphere.

If $M$ is the disjoint union of circuits $\gamma_{1}, \ldots, \gamma_{k}$ then $I N(M)$ is the join of

$$
I N\left(\gamma_{1}\right), \ldots, I N\left(\gamma_{k}\right)
$$

that is, $I N(M)=I N\left(\gamma_{1}\right) \star \cdots \star I N\left(\gamma_{k}\right)=\left\{A_{1} \cup \cdots \cup A_{k}: A_{i} \in I N\left(\gamma_{i}\right)\right.$ for $\left.1 \leqslant i \leqslant k\right\}$. The result then follows from the fact that the join of two spheres $\mathbb{S}^{k}$ and $\mathbb{S}^{l}$ is homeomorphic to the sphere $\mathbb{S}^{k+l+1}$. [13, Chapter 2.2.2]

The matroids with pairwise disjoint cycles have a nice characterization in terms of excluded minors.

Lemma 6.7. A matroid $M$ contains two circuits with non empty intersection if and only if $U_{1,3}$ is a minor of $M$.

Proof. First suppose that $M$ contains two intersecting circuits $\gamma$ and $\delta$ which intersect at $e$. Let $c \in \gamma-\delta$ and $d \in \delta-\gamma$. Restricting to $\gamma \cup \delta$ and then contracting every element except for $c, d$, and $e$, we obtain $U_{1,3}$ as a minor.

To show the converse consider any matroid $N$ and an element $e \in E$. Notice that every circuit of $N \backslash e$ is a circuit of $N$; and if $\gamma$ is a circuit of $N$, then either $\gamma$ or $\gamma \cup e$ is a circuit of $N$. It follows that if either $N \backslash e$ or $N / e$ have two overlapping circuits, so does $N$. Since $U_{1,3}$ has two overlapping circuits, so does every matroid containing it as a minor.

Now we are ready to prove our main topological result.

Theorem 1.5. Let $M$ be a matroid and < be a linear order on its ground set. The reduced external activity complex $\operatorname{Act}_{<}^{\bullet}(M)$ is contractible if $M$ contains $U_{1,3}$ as a minor, and a sphere otherwise. 
Proof. Notice that if $M$ has a coloop $c$, then both $c$ and $\bar{c}$ are cone points of $\operatorname{Act}_{<}(M)$, and are invisible in $\operatorname{Act}_{<}^{\bullet}(M)$. Therefore we may assume that $M$ is coloop free.

Let $r$ be the rank of $M$, and let $d=\operatorname{dim}\left(\operatorname{Act}_{<}^{\bullet}(M)\right)=\operatorname{dim}\left(\operatorname{Act}_{<}(M)\right)-|A E(M)|=$ $n+r-1-|A E(M)|$. We consider two cases.

1. If $M$ is not the disjoint union of circuits, $|A E(M)|<n$ by Lemma 6.4 , so $d>r-1$. Clearly $h_{d}\left(\operatorname{Act}_{<}^{\bullet}(M)\right)=h_{d}\left(\operatorname{Act}_{<}(M)\right)$, Theorem 1.4 gives $h_{d}\left(\operatorname{Act}_{<}(M)\right)=h_{d}(I N(M))$, and since $I N(M)$ is $(r-1)$-dimensional, $h_{d}(I N(M))=0$. Therefore, by Theorem 2.11, $\operatorname{Act}_{<}^{\bullet}(M)$ is contractible.

2. If $M$ is the disjoint union of circuits, then $\operatorname{Act}_{<}^{\bullet}(M) \cong I N(M)$ is a sphere invoking Proposition 6.5 and Lemma 6.6.

The result follows from Lemma 6.7.

We conclude that the simplicial complex $\operatorname{Act}_{<}^{\bullet}(M)$ is a model for a matroid $M$ which is topologically simpler than the "usual" model $I N(M)$.

\section{Questions}

- There should be "affine" analogs of the results of this paper. Geometrically, they should correspond to taking the closure of an affine subspace $L$ of $\mathbb{A}^{n}$ in $\left(\mathbb{P}^{1}\right)^{n}$, as opposed to a linear subspace, as explained in [2]. To a morphism of matroids $M \rightarrow M^{\prime}$, one may associate an external activity complex $\operatorname{Act}_{<}\left(M \rightarrow M^{\prime}\right)[2]$ and active orders $<_{\text {int }},<_{\text {ext }},<_{\text {ext } / \text { int }}$ [14]. The analogous foundational results, such as Theorems 2.4, 2.5, 2.6, 2.7 hold there as well. [1, 14] Do our main theorems hold in that more general setting?

- Even though $\operatorname{Act}_{<}(M)$ only pays attention to the external activities of the bases of $M$, it is the external/internal order $<_{\text {ext } / \text { int }}$ which plays a crucial role in its shelling. This makes the following question from [2] even more natural: is $\operatorname{Act}_{<}(M)$ part of a larger (and well-behaved) simplicial complex which simultaneously involves the internal and external activities of the bases of M? Ideally we would like it to come from a natural geometric construction.

- Notice that for an ordered matroid $M$, every linear extension of the poset of restriction sets of the lexicographic shelling order of $I N(M)$ gives another shelling order with the same restriction sets. That means that every posible order of the facets that could give a shelling with the same restriction sets gives another shelling of $I N(M)$. Does this property say something more about the independence complex. Is there a wide class of examples of a shellable complex with a fix shelling order, such that every linear extension of the poset is again a shelling. Notice that 3.2 is an example that $\operatorname{Act}_{<}(M)$ with the associated lexicographic shelling does not have this property.

\section{Acknowledgments}

The first author would like to thank Adam Boocher; this project would not exist without our collaboration in [2], and the numerous conversations with him on this topic. The 
second author would like to Isabella Novik for the invitation to the University of Washington, where part of this project was carried on. This project started at the Encuentro Colombiano de Combinatoria (ECCO 14) and we would like to thank the SFSU-Colombia Combinatorics Initiative and the Universidad de Los Andes for hosting and funding the event.

\section{References}

[1] F. Ardila. Semimatroids and their Tutte polynomials. Revista Colombiana de Matemáticas, 41:39-66, 2007.

[2] F. Ardila and A. Boocher. Closures of Linear Spaces. arXiv:1312.6874, December 2013.

[3] Federico Ardila and Caroline J. Klivans. The Bergman complex of a matroid and phylogenetic trees. J. Combin. Theory Ser. B, 96(1):38-49, 2006.

[4] A. Björner. The homology and shellability of matroids and geometric lattices. In Matroid applications, volume 40 of Encyclopedia Math. Appl., pages 226-283. Cambridge Univ. Press, Cambridge, 1992.

[5] Thomas Brylawski and James Oxley. The Tutte polynomial and its applications. In Matroid applications, volume 40 of Encyclopedia Math. Appl., pages 123-225. Cambridge Univ. Press, Cambridge, 1992.

[6] Tom Brylawski. The broken-circuit complex. Trans. Amer. Math. Soc., 234(2):417433, 1977.

[7] H. Crapo. The Tutte polynomial. Aequationes Math., 3:211-229, 1969.

[8] Jesús A. De Loera, Yvonne Kemper, and Steven Klee. $h$-vectors of small matroid complexes. Electron. J. Combin., 19(1):\#P14, 2012.

[9] Jon Folkman. The homology groups of a lattice. J. Math. Mech., 15:631-636, 1966.

[10] June Huh. Milnor numbers of projective hypersurfaces and the chromatic polynomial of graphs. J. Amer. Math. Soc., 25(3):907-927, 2012.

[11] June Huh and Eric Katz. Log-concavity of characteristic polynomials and the Bergman fan of matroids. Math. Ann., 354(3):1103-1116, 2012.

[12] Steven Klee and Jose Alejandro Samper. Lexicographic shellability, matroids and pure order ideals, 2014.

[13] D. Kozlov. Combinatorial algebraic topology, volume 21 of Algorithms and Computation in Mathematics. Springer, Berlin, 2008.

[14] M. Las Vergnas. Active orders for matroid bases. European J. Combin., 22(5):709721, 2001. Combinatorial geometries (Luminy, 1999).

[15] Criel Merino. The chip firing game and matroid complexes. In Discrete models: combinatorics, computation, and geometry (Paris, 2001), Discrete Math. Theor. Comput. Sci. Proc., AA, pages 245-255 (electronic). Maison Inform. Math. Discrèt. (MIMD), Paris, 2001. 
[16] Criel Merino, Steven D. Noble, Marcelino Ramírez-Ibáñez, and Rafael VillarroelFlores. On the structure of the $h$-vector of a paving matroid. European J. Combin., 33(8):1787-1799, 2012.

[17] Suho Oh. Generalized permutohedra, $h$-vectors of cotransversal matroids and pure O-sequences. Electron. J. Combin., 20(3):\#P14, 2013.

[18] Peter Orlik and Louis Solomon. Combinatorics and topology of complements of hyperplanes. Invent. Math., 56(2):167-189, 1980.

[19] J.G. Oxley. Matroid theory. Oxford Science Publications. The Clarendon Press Oxford University Press, New York, 1992.

[20] J. Scott Provan and Louis J. Billera. Decompositions of simplicial complexes related to diameters of convex polyhedra. Math. Oper. Res., 5(4):576-594, 1980.

[21] Gian-Carlo Rota. On the foundations of combinatorial theory. I. Theory of Möbius functions. Z. Wahrscheinlichkeitstheorie und Verw. Gebiete, 2:340-368 (1964), 1964.

[22] Gian-Carlo Rota. Combinatorial theory, old and new. In Actes du Congrès International des Mathématiciens (Nice, 1970), Tome 3, pages 229-233. Gauthier-Villars, Paris, 1971.

[23] Jay Schweig. On the $h$-vector of a lattice path matroid. Electron. J. Combin., 17(1):\#N3, 2010.

[24] Richard P. Stanley. Cohen-Macaulay complexes. In Higher combinatorics (Proc. NATO Advanced Study Inst., Berlin, 1976), pages 51-62. NATO Adv. Study Inst. Ser., Ser. C: Math. and Phys. Sci., 31. Reidel, Dordrecht, 1977.

[25] Bernd Sturmfels. Solving systems of polynomial equations, volume 97 of CBMS Regional Conference Series in Mathematics. Published for the Conference Board of the Mathematical Sciences, Washington, DC; by the American Mathematical Society, Providence, RI, 2002.

[26] W. T. Tutte. A contribution to the theory of chromatic polynomials. Canadian J. Math., 6:80-91, 1954.

[27] Hassler Whitney. A logical expansion in mathematics. Bull. Amer. Math. Soc., 38(8):572-579, 1932. 